\title{
Improvement of an Artificial Neural Network Model using Min- Max Preprocessing for the Prediction of Wave-induced Seabed Liquefaction
}

\author{
Deaho Cha, Michael Blumenstein, Hong Zhang, and Dong-Sheng Jeng
}

\begin{abstract}
In the past decade, artificial neural networks (ANNs) have been widely applied to the engineering problems with a complicated system. ANNs are becoming an important alternative option for solving problems in comparison to traditional engineering solutions, which are usually involved in complicated mathematical theories. In this study, we apply an ANN model to the wave-induced seabed liquefaction problem, which is a key issue in the area of coastal and ocean engineering. Furthermore, we adopted an ANN model with preprocessing (MIN-MAX) on difficult training data. This paper demonstrates the capacity of the proposed ANN model using MIN-MAX pre-processing to provide coastal engineers with another effective tool to analyse the stability of seabed sediment.
\end{abstract}

\section{INTRODUCTION}

From the engineering perspective, Artificial Neural Networks (ANNs) have still not been fully explored in a number of areas and deserve further investigation. During the last decade, ANNs have been applied to various engineering fields, such as prediction of rainfall intensity [1], generation of wave parameters based on hydrodynamic data [2], tide-forecasting [3], prediction of settlement of shallow foundations [4], modeling confinement efficiency of reinforced concrete [5] and wave predictions [6].

Figure 1 illustrates the change of a seabed due to wave action (Fig 1(a): Without liquefaction, Fig 1(b) With liquefaction) The phenomenon of ocean waves propagating over a porous seabed is an important concern in the design procedure of coastal offshore structures. When gravity waves propagate over the ocean, variations of wave pressure induce variations in effective stresses and pore water pressure within the seabed. With excess pore-pressure and diminishing vertical effective stress, part of the seabed may become unstable or liquefied. Furthermore, when liquefaction occurs, the soil particles are likely to be carried away as heavy fluid by any prevailing bottom currents or mass transport due to the wave action (Fig 1(b)). Many

Deaho Cha is with the Griffith School of Engineering, Griffith University Gold Coast Campus Australia

Michael Blumenstein, is with the School of Information and Communication Technology, Griffith University Gold Coast Campus Australia. (corresponding author phone: +61 7555 28271; fax: +61 7555 28066; e-mail: M.Blumenstein@griffith.edu.au).

Hong Zhang is with the Griffith School of Engineering, Griffith University Gold Coast Campus Australia.

Dong-Sheng Jeng, is with the Department of Civil Engineering, University of Sydney Australia researchers have studied solutions for wave-induced seabed liquefaction using numerical/analytical solutions and some of experimental lab work [7-10]. However, most previous investigations for wave-induced liquefaction of seabed have been based on various assumptions of engineering mechanics. The applications of the model in realistic engineering problems are limited.

In this study, we apply an ANN model, adopting a necessary pre-processing procedure on the training data for the prediction of wave-induced seabed liquefaction, which is a very critical issue in the coastal engineering field, such as for coast and coastal structures, coastal environment protection

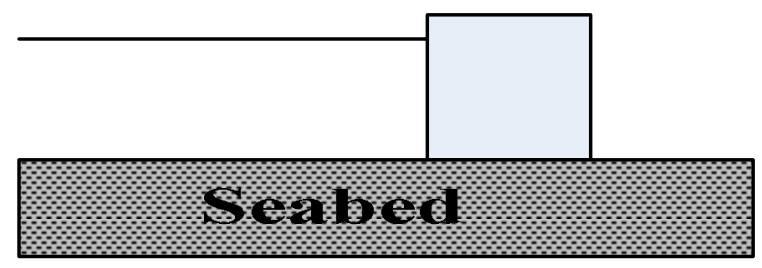

(a) Seabed without liquefaction

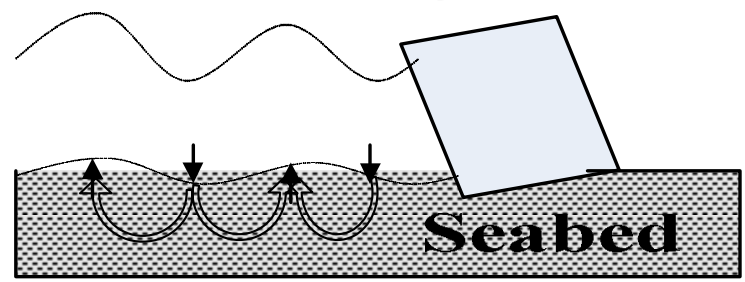

(b) Seabed with liquefaction occurs.

Fig. 1. Phenomenon of wave-induced liquefaction.

\section{ANN MODEL USING MIN-MAX PRE-PROCESSING}

Besides the use of standard ANNs for wave-induced liquefaction, we will discuss ANN models, adopting a preprocessing procedure on the training data. Generally, the ANN models training input and target data are manually acquired for prediction, which are normalised between 0 and 1 or -1 and 1 . One of the major matters for consideration during the ANN training procedure is over-fitting. Overfitting occurs when the error on the training data set is driven to a very small value, but when new data is presented to the network the error is large, hence, the network has memorised the training data but it has not learned to generalise to new 
situations. Aside from experimenting with the stopping criteria of the ANN, another possible approach for ensuring optimal training is to use an appropriate pre-processing procedure on the given data set, which can save data preparation time and facilitate the creation of a more precise data set (Figure 2).

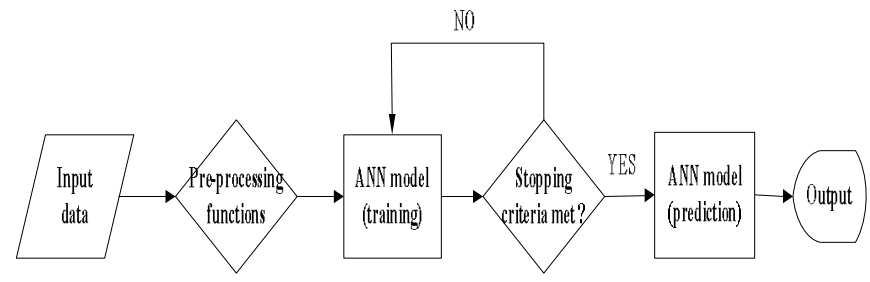

Fig. 2. Pre-processing set-up for training procedure

There are numerous pre-processing functions that may assist in optimal network training. Two such techniques are described here, which have relevance to the anomalies inherent in data associated with wave-induced seabed liquefaction. The first one normalise the inputs and targets so that they have means of zero and standard deviations of 1 , defined as Mean and Standard Deviation Functions

$$
P_{n}=\frac{\left(P_{i}-M\right)}{S_{t}}
$$

where $P_{n}$ is the pre-processed input data, $P_{i}$ is the original input data, $M$ is the mean value for $P_{i}$, and $S_{t}$ is the standard deviations for $P_{i}$

The second function is also used to normalise the inputs and targets so that they fall in the interval $[-1,1]$. It is denoted as Min and Max Functions [11]

$$
P_{n}=\frac{2 \times\left(P_{i}-P_{\min }\right)}{\left(P_{\max }-P_{\min }\right)}-1
$$

where $P_{\min }$ are the minimum values for each of the target data and $P_{\max }$ are the maximum values for each of the target data.

In this study we investigated the use of Min and Max Functions (3) for processing the training data to improve network training.

\section{A. Preparing Training and Test data sets}

In present study, we use two-dimensional wave-seabed interaction problem, and the porous seabed is treated as hydraulically isotropic with the same permeability. Biot [12] introduced general governing equations for the behaviour of a linear elastic porous solid under dynamic conditions. They are summarized in the tensor form as below

$$
\begin{aligned}
& \sigma_{i j, j}=\rho \ddot{u}_{i}+\rho_{f} \ddot{w}_{i} \\
& -p_{, i}=\rho_{f} \ddot{u}+\frac{\rho_{f}}{n} \ddot{w}_{i}+\frac{\rho_{f} g}{k_{z}} \dot{w}_{i} \\
& v_{i j}+w_{i i}=-\frac{n}{k_{f}} \dot{p}
\end{aligned}
$$

where the subscripts "i, $\mathrm{j}$ " denote the derivation with respect to the $\mathrm{i}$ - and $\mathrm{j}$ - directions, respectively, $p$ is the pore pressure, $u$ and $w$ are the displacements of solid and relative displacements of solid and fluid, $1 / \mathrm{k}_{\mathrm{f}}$ is the compressibility of pore fluid, which is defined by

$$
\frac{1}{k_{f}}=\frac{1}{2 \times 10^{9}}+\frac{1-S_{r}}{P_{w o}}
$$

where $S_{r}$ is the degree of saturation, $P_{w o}$ is the absolute water pressure. The definition of effective stress $\sigma_{i j}^{\prime}$, which is assumed to control the deformation of the soil skeleton, is given for the total stress $\sigma_{i j}$ as $\sigma_{i j}=\sigma_{i j}^{\prime}-\delta_{i j} p$.

Based on these equations we can calculate the waveinduced soil and fluid displacements, and further obtain the wave-induced pore pressure, effective stresses and shear stresses. Detailed information of the above solution can be found in [13].

It has generally been accepted that when the vertical effective stress vanishes, the soil will be liquefied. Thus, the soil matrix loses its strength to carry and load, and consequently causes seabed instability.

Based on the concept of excess pore pressure, Zen and Yamazaki proposed a criterion of liquefaction [14],

$-\frac{1}{3}\left(1+2 K_{o}\right)\left(\gamma_{s}-\gamma_{w}\right) z+\left(P_{b}-p\right) \leq 0$

where $K_{o}$ is the coefficient of earth pressure at rest, which is normally varied from 0.4 to 1.0 , and 0.5 is commonly used for marine sediments [15]. In (8), $\gamma_{\mathrm{s}}$ is the unit weight of soil, $\gamma_{\mathrm{w}}$ is the unit weight of water, and $P_{b}$ is the wave pressure at the seabed surface, which is given by

$$
P_{b}(x, t)=\frac{\gamma_{w} H}{2 \cosh k d} \cos (k x-\omega t)
$$

where, $H$ is the wave height, $k$ is the wave number, $t$ is time, $\omega$ is the wave frequency and $d$ is the water depth.

Based on these solutions we collected liquefaction depths with various soil and water characteristics. Details are discussed in the results section.

\section{SIMULATIONS AND RESULTS}

\section{A. Application of ANN models for Wave-induced Liquefaction}

Generally, it is imperative that ANN models are presented with appropriate input data and accurate values for the desired outputs. The use of ANNs is a most attractive alternative compared with traditional engineering solutions, which require solve the complicated nonlinear mathematical equations, as in the case of predicting wave-induced seabed liquefaction.

The configuration of the ANN model used in this study is shown in Figure 3. 


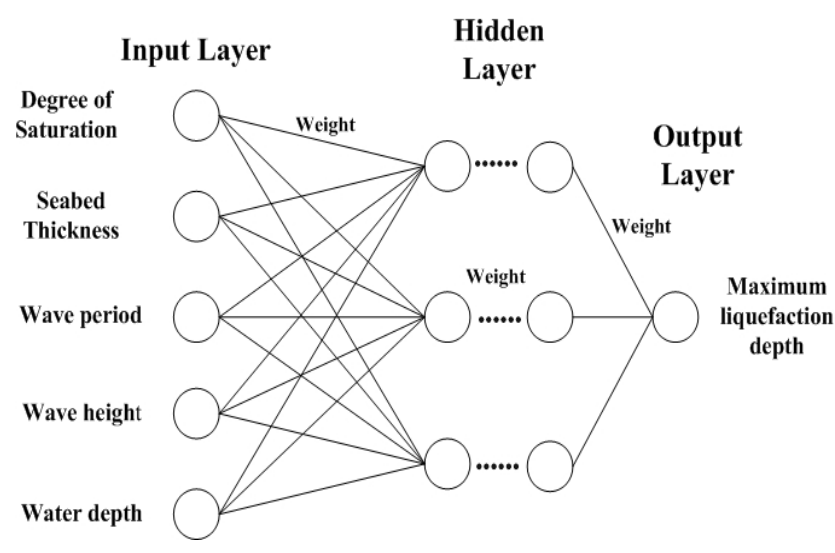

Fig. 3. Structure of an ANN model for wave-induced liquefaction.

The ANN used in this research is a standard feed-forward multi-layer perception trained using the resilient backpropagation algorithm. The performance of back propagation neural network models depends on the number of hidden layers, hidden neurons, the learning factors, the number of weight configurations and the number of neurons in each layer. The ANN configurations used in the present ANN model are presented in Table I.

TABLE I

\section{INPUT DATA OF ANN MODEL}

\begin{tabular}{|c|c|}
\hline Number of input neurons & 5 \\
\hline Number of Output neurons & 1 \\
\hline Number of hidden layers & Varied between 1 to 4 \\
\hline Number of hidden neurons & Varied between 2 to 20 \\
\hline Learning rate & Varied between 0.2 to 0.8 \\
\hline Momentum factor & Varied between 0.2 to 0.8 \\
\hline
\end{tabular}

As seen in Figure 3, we used 5 input parameters to the ANN model, which are the degree of saturation, the seabed depth, the wave period, the wave height, and the water depth. Investigations have concluded that soil permeability is very sensitive to the occurrence of the wave-induced liquefaction potential [16]. According to a previous study [16], permeabilities are very sensitive and make the procedure of normalisation quite difficult. After preliminary tests of the ANN models, we found that inclusion of permeability causes difficulties for convergence of the model. Thus, we have an ANN model for each case with a fixed value of soil permeability. This ensures that the proposed ANN model can more easily converge.

\section{B. Numerical Simulations}

We introduce an ANN model using MIN-MAX preprocessing for the data set, as well as a standard ANN model without pre-processing. The input data for the analytical results, which establishes the database of both models, is described in Table II
TABLE II

INPUT DATA OF THEORETICAL MODEL

\begin{tabular}{|c|c|}
\hline \multicolumn{2}{|c|}{ Soil Characteristics } \\
\hline Soil Permeability & $1 * 10^{-3}, 1 * 10^{-4} \mathrm{~m} / \mathrm{sec}$ \\
\hline Seabed Thickness & Varying between $10 \mathrm{~m}$ to $60 \mathrm{~m}$ \\
\hline Shear Modulus & $10^{7} \mathrm{~N} / \mathrm{m}^{2}$ \\
\hline Poisson's Ratio & 0.4 \\
\hline Porosity & 0.4 \\
\hline Degree of Saturation & Varying between 0.95 to 1.0 \\
\hline \multicolumn{2}{|c|}{ Wave Characteristics } \\
\hline Wave Period & Varying between $8 \mathrm{sec}$ to $20 \mathrm{sec}$ \\
\hline Wave Height & Varying between $0.8 \mathrm{~m}$ to $10 \mathrm{~m}$ \\
\hline Water Depth & Varying between $20 \mathrm{~m}$ to $80 \mathrm{~m}$ \\
\hline
\end{tabular}

As seen in the Table II, it covers a great range of wave and soil conditions for the training and test database. As the analytical test indicates, liquefaction occurs in various situations. In the simulations, we had approximately 20,000 data items for the liquefaction depth from each analytical model. Among this data set we used $70 \%$ for the training data and the remainder was used for verifying the prediction capacity of both ANN models.

1) Artificial Neural Network without Pre-processing: The first model attempted, was to establish a neural network without pre-processing the training data. The test results for prediction of the wave-induced seabed liquefaction depth are presented in Figure 4.

In this test, a database is generated from an analytical model with various parameters (Table II). However, the ANN model has a limitation of the input and output values between 0 and 1 or -1 and 1 . Therefore it was necessary to convert the input and output values to suit this range by manually.

In this paper, we not only use a correlation value $\left(\mathrm{R}^{2}\right)$ for comparison of the analytical model and the ANN model but we also use the Root Mean Square Error (RMSE) value. The RMSE is defined as

$R M S E=\sqrt{\frac{1}{N} \sum_{i=1}^{N}\left(L_{A}-L_{P}\right)^{2}}$

where $\mathrm{L}_{\mathrm{A}}$ and $L_{P}$ are the liquefaction depth from the ANN model and the analytical model respectively; $N$ is the total number of liquefaction depth data.

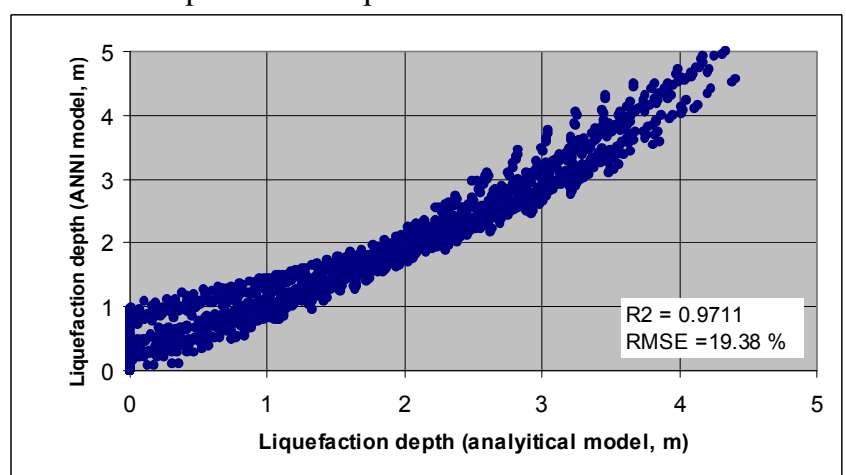

(a) Prediction of seabed liquefaction depth for the ANN model without preprocessing versus the analytical model (Soil permeability $=10^{-4} \mathrm{~m} / \mathrm{sec}$ ) 


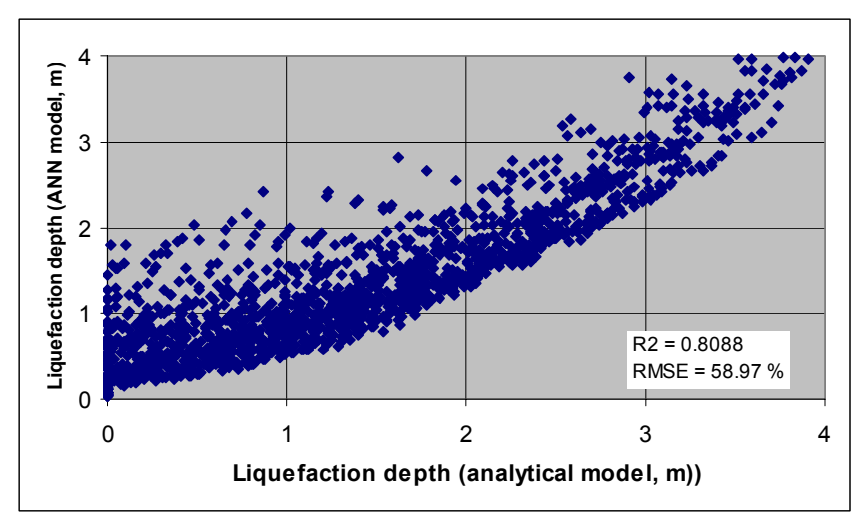

(b) Prediction of seabed liquefaction depth for the ANN model without preprocessing data versus the analytical model (Soil permeability $=10^{-3} \mathrm{~m} / \mathrm{sec}$ )

Fig. 4 Comparison of the wave-induced seabed liquefaction depth using the ANN model without preprocessing versus the analytical model (soil permeability: $10^{-4} \mathrm{~m} / \mathrm{sec}$.(a), $10^{-3} \mathrm{~m} / \mathrm{sec}$.(b)

Figure 4 (a) shows a comparison between the predicted seabed liquefaction depth using the ANN model (without pre-processing the training data) and the analytical model with soil permeability of $10^{-4} \mathrm{~m} / \mathrm{sec}$. As seen in this figure, the prediction values of the ANN model agree with the analytical model data overall with an $\mathrm{R}^{2}=0.97$ and RMSE $=$ $19.38 \%$. However, the ANN model (without pre-processing) prediction values did not agree with the analytical model when a soil permeability of $10^{-3} \mathrm{~m} / \mathrm{sec}$ for seabed liquefaction was used (Fig. 4(b)). The reason for the low performance of permeability in the $10^{-3} \mathrm{~m} / \mathrm{sec}$ case is that this particular training data set contained seabed liquefaction depth values, which were too closely concentrated within the designated range compared with those in the $10^{-4} \mathrm{~m} / \mathrm{sec}$. case. This anomaly in the training data prompted the investigation of an appropriate preprocessing procedure.

\section{2) Artificial Neural Network using Pre-Processed Data:}

The occurrence of wave-induced liquefaction depends on soil and wave parameters. However, as shown in Figure 4(b), the ANN model without pre-processing fails to predict the seabed liquefaction depth adequately due to the nonuniformity of the data. Therefore, to solve this problem, the data was pre-processed with the procedure described in equation (3) previously.

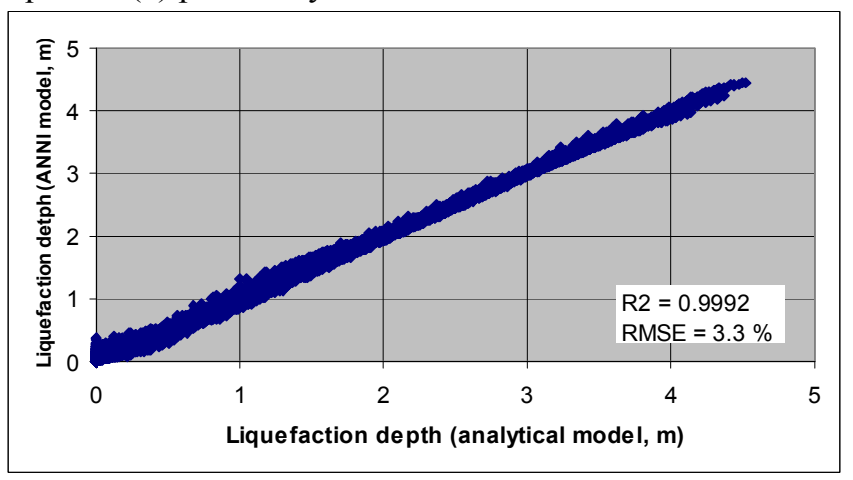

(a) Prediction of seabed liquefaction depth for the ANN model with preprocessed data versus the analytical model (Soil permeability $=10^{-4} \mathrm{~m} / \mathrm{sec}$ )

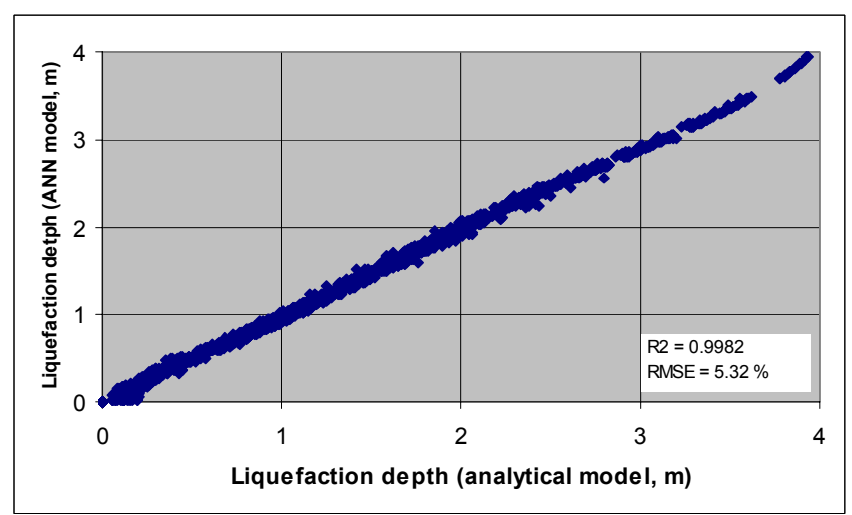

(b) Prediction of seabed liquefaction depth for the ANN model with preprocessed data versus the analytical model (Soil permeability $=10^{-3} \mathrm{~m} / \mathrm{sec}$ )

Fig. 5 Comparison of the wave-induced seabed liquefaction depth with an ANN model using Min-Max preprocessing versus the analytical model (soil permeability : $10^{-4} \mathrm{~m} / \mathrm{sec}$.(a), $10^{-3} \mathrm{~m} / \mathrm{sec}$.(b)

Figure 5 clearly indicates that the prediction performance of the ANN model using pre-processed data is significantly better than the standard ANN model (Fig 4 (a), (b)), for both cases of soil permeability.

TABLE III

RMSE AND R ${ }^{2}$ FOR ANN MODEL (WITH AND WITHOUT PRE-

PROCESSIGN OF TRAINING DATA)

\begin{tabular}{|c|c|c|c|}
\hline \multicolumn{4}{|c|}{ Soil permeability $=10^{-4} \mathrm{~m} / \mathrm{sec}$} \\
\hline \multicolumn{3}{|c|}{ ANN } & ANN with Pre-processing \\
\hline $\mathrm{R}^{2}$ & RMSE & $\mathrm{R}^{2}$ & RMSE \\
\hline 0.9711 & $19.38 \%$ & 0.9992 & $3.3 \%$ \\
\hline \multicolumn{4}{|c|}{ Soil permeability $=10^{-3} \mathrm{~m} / \mathrm{sec}$} \\
\hline \multicolumn{3}{|c|}{ ANN } & ANN with Pre-processing \\
\hline $\mathrm{R}^{2}$ & RMSE & $\mathrm{R}^{2}$ & RMSE \\
\hline 0.8088 & $53.97 \%$ & 0.9982 & $5.32 \%$ \\
\hline
\end{tabular}

For the current investigation, the results clearly indicate that the ANN model, which includes an additional step to pre-process the training/test data, can handle even the difficult, non-uniform data that is associated with waveinduced sea-bed liquefaction. The $\mathrm{R}^{2}$ and RMSE values, with different soil permeability, are summarized in Table III.

\section{CONCLUSIONS}

In this study we established an ANN model with a preprocessing stage for application to the wave-induced seabed liquefaction problem in coastal engineering. The results of this paper clearly show that the ANN model which uses preprocessed training/testing data provides better prediction performance for the wave-induced seabed liquefaction depth in both soil permeability cases that were tested as compared with that of the standard ANN model.

Unlike traditional engineering mechanics approaches, ANNs do not require a complicated mathematical procedure. It is for this reason that ANNs can be powerful engineering tools for future research. 


\section{REFERENCES}

[1] M. French, F. Witol, F. W. Krajewski, and R. R. Cuykendallb, "Rainfall forecasting in space and time using a neural network" Journal of Hydrology, 137, pp. 1-31,1992.

[2] B .Yonas, A.W.M. Dibike, and M.B. Abbott, "Applications of artificial neural networks to the generation of wave equations form hydraulic data", Journal of Hydraulic Research, vol 37 no 1, pp. 8197, 1999.

[3] T.L. Lee, and D.S. Jeng, "Application of artificial neural networks in tide-forecasting", Ocean Engineering, vol 29, pp. 1003-1022, 2002.

[4] C.W. Tang, H.J. Chen and T. Yen, "Modeling confinement efficiency of reinforced concrete columns with rectilinear transverse steel using artificial neural networks," Journal of Structural Engineering, ASCE, vol 129, pp. 775-783, 2003.

[5] . A.S. Mohamed, H.R. Mailer, M.B. Jaksa, "Prediction settlement of shallow foundations using neural networks," Journal of Geotechnical and Geoenvironmental Engineering, ASCE, vol 128, pp. 785-793, 2002.

[6] O. Makarynskyy, "Improving wave predictions with artificial neural networks", Ocean Engineering, 31, pp. 709-724, 2004.

[7] J. Bjerrum, "Geotechnical problem involved in foundations of structures in the North Sea", Geotechnique, vol 23, no 3, pp. 319-358, 1973.

[8] M.S. Rahman, "Instability and movement of ocean floor sediments. A review", International Journal of Offshore and Polar Engineering, vol. 7, no 3, pp. 220-225, 1997.

[9] S. Sassa, and H. Sekiguchi, "Analysis of wave-induced liquefaction of sand beds", Geotechnique, vol 51, no 2, pp. 115-126, 2001.

[10] S. Sassa, H. Sekiguchi, and J. Miyamamoto, "Analysis of progressive liquefaction as moving-boundary problem", Geotechnique, vol 51, no 10, pp. 847-857, 2001.

[11] D. Howard and B. Mark, "Neural Network Toolbox User Guide," The Mathwork, 2004

[12] M. A. Biot, "Theory of propagation of elastic waves in a fluidsaturated porous solid. Part I: Low frequency range; Part II. High frequency analysis". Journal of Acoustics Society, 28:168-191, 1956

[13] D.H. Cha, "Mechanism of Ocean Waves Propagating over a Porous Seabed," MPhil Thesis, Griffith University, Australia, 2003

[14] G. R. Faulhaber, "Design of service systems with priority reservation," in Conf. Rec. 1995 IEEE Int. Conf. Communications, pp. 3-8.

[15] R. F. Scott, "Principles of Soil Mechanics", Addison-Publishing, Massachusetts, 1968

[16] D. S. Jeng, "Wave-induced seabed instability in front of a breakwater". Ocean Engineering, vol 24, no 10, pp. 887-917, 1997. 